\title{
Percepção de cuidados paliativos dos profissionais de saúde no ambiente hospitalar do município de Pinheiro-MA
}

\section{Perception of palliative care of health professionals in the hospital environment of the municipality of Pinheiro-MA}

\author{
Raissa da Silva Santos ${ }^{*}$ \\ Orcid: https://orcid.org/0000-0001-7806-2429
}

Ivana Santos Vale ${ }^{3 *}$

Orcid: https://orcid.org/0000-0003-1102-5716

Bárbara Fernanda Melo Barros ${ }^{\text {* }}$

Orcid: https://orcid.org/0000-0001-5651-6629
Amanda Namibia Pereira Pasklan ${ }^{2 *}$

Orcid: https://orcid.org/0000-0001-7193-4861

Ingrid Eliza Sousa Leitão ${ }^{*}$

Orcid: https://orcid.org/0000-0002-5769-5785

Francisco Alípio de Oliveira Santiago ${ }^{6 *}$

Orcid: https://orcid.org/0000-0002-9746-413X

Sara Fiterman Lima ${ }^{7 *}$

Orcid: https://orcid.org/0000-0003-0015-3413

\begin{abstract}
Resumo
Introdução: Os cuidados paliativos trouxeram mudanças na prática dos profissionais nos serviços de saúde, ao acrescentar o papel de cuidar do processo de morrer. No entanto, é fato que, nas graduações em saúde, a abordagem desse tema ainda é insuficiente e isso tem implicações diretas sobre o tipo de cuidado ofertado a pacientes com condições limitadoras e ameaçadoras de vida Objetivo: Avaliar a percepção de profissionais de saúde sobre os Cuidados Paliativos. Materiais e método: Estudo exploratório de abordagem qualitativa realizado com profissionais de saúde da rede hospitalar da cidade de Pinheiro, Maranhão. Participaram da pesquisa 18 profissionais, dos quais 12 enfermeiros e 6 médicos. A coleta de dados se deu por meio de entrevista semiestruturada, sendo as entrevistas gravadas e posteriormente transcritas para permitir a interpretação dos dados que seguiu o método da análise de temática em Bardin. Resultados: Os profissionais apresentaram pouco conhecimento acerca do tema, além de insegurança para lidar com a terminalidade e a garantia da autonomia do paciente. Além disso, verificou-se uma dificuldade na aceitação da morte tanto por parte da equipe como por parte do paciente e dos familiares. Conclusão: A apropriação do conhecimento dos Cuidados Paliativos é essencial para a garantia da dignidade humana. Portanto, o oferecimento de treinamento e a discussão desse tema no ambiente de trabalho é fundamental para a garantia da qualidade de vida do indivíduo.
\end{abstract}

Palavras-chave: cuidados paliativos; prestação integrada de cuidados de saúde; conhecimento. assistência hospitalar.

\section{Abstract}

Introduction: Palliative care has brought changes in the practice of professionals in health services, by adding the role of caring for the process of dying. However, it is a fact that, in health degrees, an approach to this topic is still insufficient and this has directives on the type

\footnotetext{
${ }^{1}$ E-mail: raissasantos-nt@hotmail.com

${ }^{2}$ E-mail: amanda_namibia@hotmail.com

${ }^{3}$ E-mail: ivanasvale@outlook.com

${ }^{4}$ E-mail: ingrideliza2010@hotmail.com

${ }^{5}$ E-mail: barbara.fernanda@discente.ufma.br

${ }^{6}$ E-mail: alipio.santiago@gmail.com

${ }^{7}$ E-mail: sara.fiterman@gmail.com

* Universidade Federal do Maranhão, Brasil.
} 
of care offered to patients with limiting and life-threatening conditions Objective: to assess the perception of health professionals about Care Palliative. Materials and method: Exploratory study with a qualitative approach carried out with health professionals from the hospital network in the city of Pinheiro, Maranhão. Eighteen professionals participated in the research, of which 12 nurses and 6 doctors. Data collection took place through semi-structured interviews, with the statements recorded and later transcribed to allow the interpretation of the data that followed the method of thematic analysis in Bardin. Results: Professionals with little knowledge about the topic, besides insecurity to deal with terminality and the guarantee of patient autonomy. In addition, there was a difficulty in accepting death both on the part of the team and on the part of the patient and family. Conclusion: The appropriation of the knowledge of Palliative Care is essential for the guarantee of human dignity. Therefore, the provision of training and the discussion of this topic in the work environment is essential to guarantee the individual's quality of life.

Keywords: palliative care; delivery of health care; integrated; knowledge hospital care

\section{Introdução}

A evolução da tecnologia médica trouxe inúmeros benefícios à saúde da população. Dentre os quais, há que se destacar, a obtenção de diagnósticos acurados, bem como o tratamento precoce de enfermidades de mau prognóstico ${ }^{(1)}$. Esse avanço tecnológico, associado ao desenvolvimento da terapêutica, resultaram no aumento da prevalência das doenças crônicas e da sobrevida desses pacientes. O indivíduo vulnerável, devido a sua doença, deixou de ser o centro do cuidado e passou a ser instrumentalizado objetivando a manutenção da vida ${ }^{(2,3)}$.

Nessa nova perspectiva, surgiram os cuidados paliativos (CP), buscando a assistência integral e multiprofissional do paciente até o fim da vida, a fim de minimizar seu desconforto e oferecer suporte emocional e espiritual aos seus familiares (2). Segundo Khoshnazar e colaboradores ${ }^{(4)}$, CP trata-se de um filosofia de cuidado com o objetivo de fornecer um tratamento organizado, afim de aliviar ou atenuar o sofrimento, por meio da identificação precoce, do manejo adequado da dor e outros problemas físicos, mentais e intelectuais apresentados pelos pacientes.

Os CP trouxeram ainda mudanças no papel dos profissionais nos serviços de saúde, ao acrescentar o papel de cuidar do processo de morrer. Ressalta-se que a formação dos profissionais de saúde sempre esteve voltada para aspectos biológicos, orientada pelo método tecnicista, conservando estreita relação com o modo de atenção à saúde vigente. Sua prática mostrase predominantemente individual e pautada em intervenções fragmentadas de diferentes profissionais para o mesmo paciente, a fim de oferecer à população o maior número possível de serviços de saúde ${ }^{(5,6)}$.

Dessa forma, a falta de formação no tema acaba por gerar insegurança e inefetividade na aplicação desses cuidados. Estudos demonstram que o trabalho com esses pacientes exige formação especial incluindo capacitação e atualização contínua sobre o assunto. No entanto, a realidade vigente demonstra que boa parte dos profissionais de saúde não se sente preparada para atender de forma adequada essa população ${ }^{(7)}$.

Dentre as habilidades específicas para a realização desses cuidados destacamse conhecimentos acerca dos sintomas do paciente, do controle da dor, da qualidade de vida e da morte e da interação familiar na fase final da vida. Isso tudo é essencial para ajudar o doente a lidar com as limitações inerentes a sua enfermidade ${ }^{(7)}$.

A deficiência na formação profissional acaba levando a adoção de medidas fúteis para a manutenção da vida. Em relação ao Brasil, ainda há inúmeros desafios a serem vencidos, dentre os quais destaca-se a questão da formação acadêmica. Santos e colaboradores (6) propõem em seu estudo, voltado para médicos anestesiologistas, que a formação deve buscar a valorizar a autonomia do paciente e o desenvolvimento de 
habilidades de comunicação com o paciente e familiares. Dessa forma, a terapêutica e o exercício desse cuidado tornam-se mais efetivos.

Justifica-se esse estudo devido pesquisas mostrarem que o ambiente profissional associado a esse cuidado ainda se apresenta repleto de profissionais pouco preparados e de formação inadequada para o exercício adequado dessa filosofia. A partir da investigação do conhecimento profissional sobre $\mathrm{CP}$, será possível identificar possíveis limitações na aplicação desses cuidados, no intuito de fomentar discussões e incentivar o desenvolvimento de uma rede integrada em prol de cuidados interdisciplinares, sensíveis aos desejos e necessidades do paciente e da família nesses espaços de atenção. Nesse sentido, o objetivo deste estudo foi analisar a percepção dos profissionais de saúde sobre cuidados paliativos.

\section{Materiais e Métodos}

\section{Amostra e tipo de estudo}

Foi realizada uma investigação do tipo exploratória, de abordagem qualitativa no Hospital Regional Dr. Antenor Abreu, na cidade de Pinheiro, Maranhão.

\section{Delineamento da pesquisa}

$\mathrm{O}$ número de participantes foi atingido por meio do método de "saturação teórica", compreendida como o momento em que, o incremento de novas observações não produz aumento significativo de informações. A saturação foi atingida com 18 entrevistados, destes 12 enfermeiros e 6 médicos, atuantes na rede hospitalar do município mediante assinatura de Termo de Consentimento Livre e Esclarecido.

\section{Critérios de Inclusão e Exclusão}

Foram incluídos todos os profissionais de saúde que tivessem experiência e atuação no serviço da Unidade de Terapia Intensiva de um hospital público na Baixada Maranhense. Foram excluídos do estudo aqueles profissionais que apesar de estarem atuando no setor, encontravam-se afastados por licença saúde, licença maternidade ou férias.

\section{Procedimentos}

Tais entrevistas aconteceram do mês de maio a agosto de 2019, foram armazenadas em mídia digital por meio de gravador e posteriormente transcritas na íntegra. Os dados foram analisados a partir do referencial da Análise de Conteúdo na modalidade temática proposta por Bardin ${ }^{(8)}$, onde a operacionalização da análise de conteúdo abrange três etapas: a pré-análise; a exploração do material, ou codificação; o tratamento dos resultados, a inferência e a interpretação.

Os dados encontrados foram categorizados na modalidade temática. Segundo Minayo ${ }^{(9)}$, nessa modalidade, a construção de categorias é utilizada para determinar agrupamentos de ideias sobre um determinado conceito, numa análise que consiste em descobrir os núcleos de sentido que compõem uma comunicação, cuja presença ou frequência signifiquem alguma coisa para o objetivo analítico visado.

Após a categorização, os resultados foram utilizados para a construção dos temas, ressaltando-se que, para Minayo ${ }^{\left({ }^{(9)}\right.}$, a noção de Tema está ligada a uma afirmação a respeito de determinado assunto e pode ser apresentada por uma palavra, frase ou resumo.

O projeto foi aprovado pelo Comitê de Ética em Pesquisa com Seres Humanos da Universidade Federal do Maranhão (CAAE: $\quad$ 03689218.3.0000.5087), atendendo à Resolução 466/2012 do Conselho Nacional de Saúde do Ministério da Saúde, que dispõe sobre diretrizes e normas regulamentares da pesquisa envolvendo seres humanos.

\section{Resultados}

Participaram do estudo 18 profissionais, destes 12 enfermeiros e 6 médicos. A média de idade dos participantes foi de 32 anos. O tempo médio 
de formação na área foi de 6,5 anos, já o tempo de atuação na instituição foi de 4,5 anos. Os profissionais entrevistados no ambiente hospitalar trouxeram as seguintes áreas temáticas para reflexão: relação da equipe com o paciente e aceitação.

\section{Relação da equipe com o paciente}

A equipe entrevistada apresentou diversas vertentes em relação ao conceito de CP.

\section{[...]o nosso tratamento tem que continuar, ele tem que perdurar com o objetivo de aliviar a dor desse paciente. Não só a dor física, mas também a dor espiritual, a dor psicológica, e muito mais. (M02)}

Esse depoimento foi o único que incluiu a espiritualidade dentro do conceito de CP. Os demais voltaram-se mais para a terminalidade inerente ao paciente e ao alívio do sofrimento.

Na verdade, é o cuidado com o paciente em seu estado terminal. É aquela forma de diminuir o sofrimento do paciente, que já está em seu estado terminal. A gente vai proporcionar a melhor forma do paciente chegar em sua morte, sem sofrer mais ainda. Dores, falta da família... essas coisas. (E02)

Os participantes ressaltaram a ideia de que toda a equipe deve estar envolvida na realização desse cuidado. E ainda, colocaram a necessidade de uma equipe qualificada e, principalmente humanizada.

"Eu acredito que nesse momento todos os profissionais são importantes. "(E10)

[...]Médico, que tenha amor ao próximo, porque tem uns que chegam falando coisas que acaba é piorando [...] Médico mais humano. Porque o primeiro foi horrivel. (E03)

[...]É importante que seja um médico, de preferência um médico que tenha uma visão e uma formação humanizada. (M01)

Os participantes da pesquisa mostraram que existe uma deficiência importante da abordagem desse tema na formação profissional. Apenas $61 \%$ dos participantes afirmaram ter mantido contato com o tema na graduação, apesar de se tratar de um contato superficial segundo relatos. Quando questionados sobre o contato na graduação as respostas foram:

[...]Sim, tive. Foi um assunto de uma disciplina. (E04)

[...]Foi uma cadeira de saúde mental, a professora falava muito sobre esse tema. (E05)

Ainda tivemos relatos que mostraram total desconhecimento do assunto quando questionados sobre os pacientes que deveriam receber esse cuidado. Esse relato evidencia como esse tema tem sido abordado de forma deficitária.

[...]Paciente de realização de curativo, paciente que vem fazer amostra de um exame, que não é pra fazer aqui, mas ele vem sempre fazer. Paciente que vem com pequenos ferimentos. (E01)

Podemos notar ainda, em algumas falas, que a ideia proposta pelas diretrizes aparece de forma frequente, o que nos remete ao desconhecimento do termo "Diretivas Antecipadas de Vontade" e não do conteúdo dessa ferramenta.

[...]Por mais que eu consiga fazer a última coisa para que o 
paciente viva, mas se o desejo dele for eu não intervir naquele momento, eu tenho que fazer o desejo dele. Mas, claro, se não houver nenhuma declaração por parte do cliente, que ele quer ou não que haja o procedimento, eu vou trabalhar para mantê-lo vivo. (E07)

[...]Não, com esse nome principalmente não! (M04)

Além disso, o processo de morte e morrer ainda gera muita discussão entre os profissionais. Dentre os relatos fica claro que existe uma resistência grande à aceitação da morte.

[...]de o paciente está parando na minha frente e o familiar diz: "não, não quero que faça nada, porque ele já está em fase terminal e eu não quero prolongar o sofrimento do meu pai." Então já teve algumas situações bem delicadas, que eu enquanto profissional fiquei em uma situação bem chata. Porque é complicado você olhar uma pessoa morrendo na sua frente e não poder, não é nem poder, é que você não deve fazer o que o familiar não quer [...] aí você fica em uma situação delicada, é complicado. (E07)

[...], mas é bem complicado pra equipe, a gente acaba se apegando com o paciente e a gente sabe que ele não vai ter uma melhora né? Que infelizmente ele vai evoluir a óbito. (E11)

Quando indagados se a equipe deve fazer de tudo para manter o paciente vivo tivemos respostas a favor da distanásia, mesmo indo de encontro ao desejo do paciente.
[...]De jeito nenhum. Fazer de tudo. Eu mesma faço de tudo. Nem que não queria, mas a gente tem que fazer, porque você vai conversando e ele vai olhando porque os olhos dizem tudo. (E06)

[...]é feito isso geralmente, lutar até o último momento, enquanto o paciente tá respirando, a gente fica lutando para tentar salvar aquela vida. Não pode abandonar jamais no meio do caminho. (E01)

\section{Aceitação}

O conteúdo das entrevistas traz ideias que evidenciam a forma como o paciente e a família lidam com a morte. Muitas falas refletem a difícil aceitação e do medo desse momento. Alguns profissionais trataram do assunto colocando-se no lugar do paciente, já outros não conseguiram, se quer, imaginar o que sente o paciente.

Não é uma questão de estar na condição de paciente, é uma questão de estar na condição de ser humano. Todos nós temos medo do desconhecido $e$ a morte é algo desconhecido e, por isso, todos têm muito medo. (M02)

É um assunto muito pesado, não sei nem como responder... Deve ser bem difícil aceitar que não tem mais um diagnóstico provável de vida. (E04)

[...]no início tem aquele impacto, ai depois, o que que acontece, ela vai amadurecendo, amadurecendo... aí nos últimos dias já tem uma certa aceitação[...] (M05)

Durante o luto, o indivíduo manifesta a necessidade de reviver experiências, de valorizar o que viveu e a presença dos entes queridos. A família, no entanto, parece 
apresentar maior dificuldade para aceitação da terminalidade o que pode interferir na conduta do paciente.

A família não aceita, né[...] A família até naquele último instante eles querem que a gente faça alguma coisa. (E05)

Outra questão que chama atenção é a dificuldade em aceitar a finitude da vida em pacientes jovens, como apresentado na fala abaixo:

[...],mas quando são pessoas novas, que podem se reerguer, voltar a si, é... quando eles têm mais chance de cura. Temos que fazer o máximo possivel, $e$ o impossível pra fazê-lo voltar a vida. (E09)

A fé foi citada por muitos como um instrumento de apoio para a superação desse momento. Observou-se em algumas falas a manifestação do apelo ao divino pela cura, ainda dentro do processo de negação de um prognóstico ruim.

Religião, eles se apegam muito a Deus. Acham que Deus vai cura. (E10)

As falam revelam ainda que os profissionais parecem não se sentir aptos a estabelecer um vínculo:

Quando você pergunta quem que quer morrer, ninguém quer morrer. Então você trabalhar com o paciente a ideia de que ele vai evoluir à óbito a qualquer momento é complicado. Complicado tanto para o paciente quanto para a equipe. (E07)

Um dos entrevistados, enfermeiro, relatou uma situação que vivera enquanto acompanhante:
Horrivel. Ele chegou pra minha esposa e perguntou, quando ela estava bem lúcida, conversando, se ela queria ser intubada quando ela parasse. Sem nenhuma preparação. Chegou foi perguntando: Olha, tu tá sabendo que não tem mais o que fazer por ti, vai querer ser intubada ou não? (E03)

\section{Discussão}

Quanto a área temática "Relação da equipe com o paciente", alguns poucos aproximaram-se ao conceito proposto pela OMS, no qual o objetivo é melhorar a qualidade de vida de pacientes e familiares no contexto de uma doença grave $\mathrm{e}$ ameaçadora da vida por meio da prevenção, do alívio do sofrimento, da identificação precoce e do tratamento impecável da dor e de outros sintomas e problemas físicos, psíquicos, sociais e espirituais" (10).

O sofrimento relatado pelos entrevistados não é somente associado a dor física presente na doença, mas sim um reflexo à toda a situação vivida pelo paciente, o afeta de forma intrínseca e extrínseca, e na forma como se relaciona com o mundo ${ }^{(11)}$.

Esse tema CP ainda precisa de muitos ajustes dentro da grade curricular atual. Corroborando esse resultado, um inquérito realizado na cidade de São Paulo com acadêmicos mostrou que $83 \%$ não receberam informação satisfatória sobre pacientes em situação terminal durante a graduação ${ }^{(12) .}$

Além disso, a formação profissional deveria desenvolver as competências e as habilidades específicas necessárias para a realização desse cuidado. Quanto mais especializados e treinados for a equipe, melhores são os resultados no controle da dor e do sofrimento psicossocial. Dessa forma, o investimento nesses quesitos torna-se essencial para uma assistência adequada de pacientes em CP ${ }^{(13)}$. 
É válido ressaltar que $100 \%$ dos participantes negaram já ter participado de alguma reunião que abordasse esse tema no hospital pesquisado. Além disso, todos relataram desconhecer as Diretivas Antecipadas de Vontade.

Quanto às DAV, estas funcionam como uma ferramenta para o paciente manifestar suas vontades no final de sua vida (a manifestação deve ser antes do final da vida, pois ela tem objetivo de assegurar que sua vontade foi respeitada). Dessa forma, promovem autonomia do paciente e a garantia da dignidade humana, ao passo que permitem, na ausência de capacidade de decisão do paciente, que sua vontade será conhecida e, caso haja uma procuração de cuidados de saúde, nomeia uma pessoa de confiança do outorgante para deliberar sobre suas decisões ${ }^{(14)}$.

Os profissionais da área de saúde, em especial o médico, são formados com ênfase do processo de curar. No entanto, nem sempre a cura é possível, dessa forma o profissional toma para si uma postura de negação da morte. Essa dificuldade de lidar com a morte de pacientes pode levar a consequência emocionais e dificuldades no trabalho. Dessa forma, o profissional deve ser lembrado como indivíduo com suas fraquezas e sentimentos e não de forma robotizada ${ }^{(15)}$.

Devido a isso, quanto se aborda sobre o tema distanásia, há tanta discussão. A distanásia consiste no emprego obsessivo de procedimentos terapêuticos, em pacientes na fase final de vida. Os procedimentos são aplicados sem uma real reflexão sobre sua necessidade, trazendo mais malefícios ao paciente que benefícios (16). O emprego da distanásia reside na não aceitação da morte por parte dos profissionais de saúde e familiares, que acabam se apegando a tecnologias fúteis para manter a vida do paciente. Há que se ressaltar que o emprego dessas tecnologias no fim da vida não significa a garantia de uma morte com dignidade ${ }^{(17) .}$

Quanto à área temática "Aceitação", notamos pelas falas que o tema é um assunto evitado, apesar de ser uma situação do cotidiano. Isso ocorre no intuito de criar afastamento e sentimento de impossibilidade de sua ocorrência. Essa aversão à morte reside na possibilidade de sofrimento físico, do medo da solidão, da separação, do desconhecido, da interrupção dos planos e sonhos, do futuro de quem fica, além da necessidade de rever suas prioridades e valores existenciais ${ }^{(18,19)}$.

Embora o primeiro impacto diante da certeza da morte seja de negação, o paciente pode superar seus medos e enfim alcançar a aceitação, o que não significa desistir de viver, mas sim de aprender a conviver com sua real condição. Esse fato demanda todo um processo de entendimento da situação com todas as suas consequências ${ }^{(19)}$.

O processo de luto leva o paciente a criar concepções sobre o mundo, favorecendo o enfretamento do luto, bem como reorganização de sua vida ${ }^{(11)}$.

Há que se ressaltar que os $\mathrm{CP}$ buscam oferecer apoio não só ao paciente, mas à sua família, que além de auxiliar o emocional, atinge diretamente a tomada de decisões, em especial quando o paciente tem a comunicação limitada ${ }^{(11,16)}$. Logo, a família deve estar ciente do prognóstico do paciente e ser conduzida no processo de amadurecimento do luto. Dessa forma, pode-se evitar culpabilização, distanásia ou potencialização de processos de luto complicado no ambiente familiar ${ }^{(16)}$.

A morte pode ser considerada a última etapa do ciclo vital, portanto, parece estar sempre relacionada a pacientes idosos. Ao chegarem a essa etapa da vida o esperado é que já tenham cumprido sua jornada na vida, dessa forma o fim da vida parece ser mais "aceitável" nessa fase da vida. Entretanto, a possibilidade de morte existe desde o nascimento do indivíduo ${ }^{(19)}$, mas deixa de ser aceita pois os jovens ainda têm muito o que conquistar. A equipe, a família e o paciente têm uma dificuldade maior em aceitar a morte num estágio inicial do ciclo vital ${ }^{(20)}$.

A fé ajuda na redução da ansiedade, no aumento confiança e na força interior, 
dessa forma, auxilia no enfretamento, na aceitação e na superação das adversidades inerentes à terminalidade. Apesar de muitos depositarem na figura divina a possibilidade de cura, a religiosidade auxilia na redução da ansiedade e dos medos, na manutenção da esperança e em permanecer no controle da situação. Dessa forma, colabora para a autonomia e colaboração do paciente ${ }^{(20,21)}$.

Além disso, o processo de comunicação entre os profissionais e pacientes foi bastante enfatizado. Esse processo é essencial para criar uma relação de confiança entre a equipe e o paciente, de forma que ele se sinta à vontade para manifestar seus desejos, medos e valores, no intuito de direcionar conduta terapêutica considerando sua individualidade ${ }^{(11)}$.

Mais uma vez ressalta-se a insegurança dos profissionais mediante o tema. Situações que envolvem adoecimento e morte estão associadas à dificuldade na comunicação, em especial devido a limitações pessoais dos profissionais. $\mathrm{O}$ despreparo profissional para lidar com esse momento tem cerne no déficit oriundo da formação no que diz respeito a comunicação de notícias difíceis ${ }^{(15)}$.

A comunicação é essencial para a execução dos CP. É considerada uma ferramenta indispensável, pois a partir dela o paciente pode sanar dúvidas, reduzir a ansiedade e a aflição, ser confortado e dessa forma melhorar a qualidade de vida. ${ }^{(22)}$. Trechos das falas evidenciam a falta de vínculo e empatia do profissional, o que sem dúvidas funcionou como barreira para a comunicação adequada (confuso), logo, forneceu ao entrevistado uma experiência ruim a respeito dos $\mathrm{CP}$ e dificultou ainda mais o processo de aceitação da terminalidade.

\section{Conclusão}

Os relatos desses profissionais evidenciam que existe uma carência muito grande acerca de CP no ambiente hospitalar. Os conceitos colocados pelos participantes, em sua maioria incompletos, associaram o tema à terminalidade e ao alívio do sofrimento. Nesse contexto, o entendimento encontrado girou em torno do controle dos sintomas e deixou de lado a questão espiritual, social e psíquica do paciente.

Essa carência reside na deficiência na formação desses profissionais. Uma parcela significativa dos participantes declarou não ter tido contato na graduação, ainda assim os que tiveram foram de forma insuficiente para a construção de uma base sólida. As consequências dessa má formação foram: profissionais despreparados e inseguros, e pacientes pouco colaborativos.

Além disso, o fato de nenhum profissional mostrar conhecimento sobre as DAV gera preocupação, visto que servem de instrumento para manutenção da autonomia do paciente. Fica clara a necessidade de capacitação e treinamento da equipe no intuito de garantir a execução dos CP de forma efetiva.

A morte ainda é um tema difícil de ser tratado na atualidade. Os profissionais ainda têm sua formação voltada para cura e enfrentam o fim da vida como um fracasso, e não como uma parte do ciclo vital. A desestigmatização desse tema é necessária, porém complexa. Há que se abordar, de forma leve, no cotidiano, em todos os ambientes sociais e dessa forma levar cada um de nós a refletir sobre como o enfrentaremos, independente de doença, ou prognóstico. A morte é uma certeza para todos, independente de idade, portanto merece ser discutida e desmistificada.

Além disso, um importante instrumento de enfrentamento é a fé. Muitas falas foram entremeadas desse sentimento, que parece servir de apoio para superação das adversidades, bem como redutor da ansiedade e do medo. Os profissionais, em suas falas, ressaltam que o paciente busca na fé a força que precisa para enfrentar o momento de dificuldade.

Um ponto de extrema relevância no estudo foi a importância de um processo de comunicação eficaz. A comunicação é o principal instrumento dos CP. Quando bem 
realizada permite a criação de vínculo entre a equipe e o paciente e colabora para realização do cuidado adequado. Entretanto, o que percebemos foi uma dificuldade em realizar uma comunicação efetiva, fato que reside na má formação citada anteriormente, bem como na ausência de treinamentos pautados na comunicação de notícias difíceis.

\section{Referências}

1. Dadalto L, Affonseca CA. Consideraciones médicas, éticas y jurídicas sobre decisiones de fin de la vida en pacientes pediátricos. Revista Bioética. 2018; 26:12-21.

2. Garcia JBS, Rodrigues RF, Lima SF. A estruturação de um serviço de cuidados paliativos no Brasil: relato de experiência. Revista Brasileira de Anestesiologia. 2014; 64:286-291.

3. Silveira MH, Ciampone MHT, Gutierrez BAO. Percepção da equipe multiprofissional sobre cuidados paliativos. Revista Brasileira de Geriatria e Gerontologia. 2014; 17:7-16.

4. Khoshnazar TAK, Rassouli M, Akbari ME, Lotfi-Kashani F, Momenzadeh S, Haghighat S, Sajjadi M. Structural Challenges of Providing Palliative Care for Patients with Breast Cancer. Indian Journal of Palliative Care. 2016; 22(4):459-466.

5. Porto AR, Thofehrn MB, Pai DD, Amestoy SC, Arrieira ICO, Joner LR. Visão dos profissionais sobre seu trabalho no programa de internação domiciliar interdisciplinar oncológico: uma realidade brasileira. Avances en Enfermaría. 2014; 32(1):72-79.

6. Santos MFO, Teles NO, Oliveira HJ, Gomes NC, Valkasser JC, Nóbrega EC. Avaliação do conhecimento dos anestesiologistas sobre cuidados paliativos. Revista Bioética. 2014; 22(2):373-379.

7. Silva HA, Viana GKB, Lima AKG, Lima ALA. Intervenção em cuidados paliativos: conhecimento e percepção dos enfermeiros. Revista de Enfermagem UFPE on line. 2018; 12(5):1325-1330.

8. Bardin L. Análise de conteúdo. São Paulo: Edições 70; 2011.

9. Minayo MCdS. O desafio do conhecimento: Pesquisa Qualitativa em Saúde. 5th ed. São Paulo; 1998.

10. Carvalho RT, Souza MRB, Franck EM, Polastrini RTV, Crispim D, Jales SMCP. Manual de Residência de Cuidados Paliativos Barueri: Manole; 2018.

11. Fernandes MA, Carla Braz E, Platel ICdS, Agra G, Lopes MdS, Rodrigues FdAe. Percepção dos enfermeiros sobre o significado dos cuidados paliativos em pacientes com câncer terminal. Ciência e Saúde Coletiva. 2013; 18(9):2589-2596.

12. Pinheiro TRSP. Avaliação do grau de conhecimento sobre cuidados paliativos e dor dos estudantes de medicina do quinto e sexto anos. O mundo da saúde. 2010; 34(3):320-326.

13. Cardoso DH, Muniz RM, Schwartz E, Arrieira ICO. Cuidados paliativos na assistência hospitalar: a vivência de uma equipe multiprofissional. Texto \& Contexto - Enfermagem. 2013; 22(4):1134-1141.

14. Oliveira MBd. Diretivas antecipadas de vontade: muitas questões e ainda mais respostas. 2014. FMUC Medicina - Teses de Mestrado.

15. RibeiroI JR, Poles K. Cuidados Paliativos: Prática dos Médicos da Estratégia Saúde da Família. Revista Brasileira de Educação Médica. 2019; 43(3):62-72.

16. Ferreira JMG, Nascimentos JL, Sá FC. Profissionais de saúde: um ponto de vista sobre a morte e a distanásia. Revista Brasileira de Educação Médica. 2018; 42(3):87-96. 
17. Guimarães MM, Carvalho MAF, Simões IAR, Lima RS. Eutanásia e distanásia: percepção de médicos e enfermeiros de uma cidade sul mineira. Revista de enfermagem da UERJ. 2016;24(2):e9545.

18. Santos APD, Alves PGS, Martins SES, Oliveira JA. Morte e morrer: uma perspectiva antropológica e pedagógica sobre o morrer. Formação Docente. 2018;10(2).

19. Borges ADVS, Silva EF, Mazer SM, Toniollo PB, Valle ERM, Santos MA. Percepção da morte pelo paciente oncológico ao longo do desenvolvimento. Psicologia em Estudo. 2006; 11(2):361-369.

20. Verzola ACZ, Bárbaro MMM. Os significados da morte para jovens universitários: uma leitura psicanalítica. 2009. Trabalho de Conclusão de Curso (Graduação)- Faculdades Integrada Fafibe.

21. Nobre CC, Mendes FR. A experiência da pessoa com dor oncológica na sua transcendência. Revista Ibero-Americana de Saúde e Envelhecimento. 2018.

22. Brito F, Coutinho MJF, Andrade CG, Costa SFG, Costa ICP, Santos KFO. Cuidados paliativos e comunicação: estudo com profissionais de saúde do serviço de atenção domiciliar. Cuidado é Fundamental. 2017; 9(1):215-221.

\section{Como citar este artigo:}

Santos RS, Pasklan ANP, Vale IS, Leitão IES, Barros BFM, Santiago FAO, Lima SF. Percepção de cuidados paliativos dos profissionais de saúde no ambiente hospitalar do município de Pinheiro-MA. Rev. Aten. Saúde. 2021; 19(69): 363-372. 